\title{
Knowledge, Attitudes, Perceptions and Practices Related to Ovicidal-Larvicidal Traps for Dengue Control among Households in One Barangay in Quezon City
}

\author{
Joanah Celine R. Felizardo, Guian Carlo C. Labo, Louise Gabriella M. Valdez, \\ Jerome E. Villacorta and Ernesto R. Gregorio, Jr. \\ Department of Health Promotion and Education, College of Public Health, University of the Philippines Manila
}

\begin{abstract}
Objective. The study aimed to establish the knowledge, attitudes, perceptions and practices of households in Quezon City on the novel dengue Ovicidal-Larvicidal (OL) traps.

Methodology. This is a descriptive cross-sectional study done in one barangay in Quezon City. This barangay was purposively chosen for two reasons: 1) it has a high incidence of dengue and, 2) it is one of the recipient barangays of DOST-developed $\mathrm{OL}$ traps distributed by $\mathrm{DOH}$. Data were collected through face to face interview using a pilot-tested interview schedule and observation. Respondents were also purposively included if they were a resident of the barangay, at least 13 years old, have received an OL trap \& instructions, and gave consent. A total of 399 respondents were recruited which was more than the computed sample size of 383.
\end{abstract}

Results. Of the 399 respondents, $92 \%$ were females, age ranged from 13 to 82 (mean of 39), 85\% were married and 54\% finished secondary education. Majority had high level of knowledge on OL traps components, properties, installation, and maintenance. Generally, $65 \%$ to $90 \%$ of respondents had favorable attitudes and perception towards dengue OL traps. Some misconceptions like OL trap is not safe to human and that it can kill mosquitoes even without adding the pellets were found.

Conclusions. The study generally established good knowledge, positive attitudes and perceptions and correct practices among respondents. Misconceptions need to be corrected to increase the acceptability of using the OL traps at the household level.

Key Words: Dengue fever, ovicidal-larvicidal trap, knowledge, attitudes, perceptions and practices

\section{Introduction}

Dengue Fever (DF) is a viral disease characterized by sudden onset of fever, headache, rashes, muscle and joint pain. It becomes fatal when it progresses to Dengue

Corresponding author: Ernesto R. Gregorio, Jr. MPH

Department of Health Promotion and Education

College of Public Health

University of the Philippines Manila

625 Pedro Gil Street, Ermita, Manila 1000 Philippines

Telephone: +6325260811

Email: junr.gregorio@yahoo.com
Hemorrhagic Fever (DHF). In the Philippines, the infected day-biting female Aedes aegypti mosquito transmits this viral infection to humans. The Department of Health (DOH) Disease Surveillance Report from September 1 to 13, 2013 showed that there were 117,658 dengue cases with 433 deaths reported giving a Case Fatality Rate (CFR) of $0.37 \%$. The age range of dengue cases was from less than 1 month to 98 years old (median $=12.83$ years). Majority $(53 \%)$ of cases were males while more than a third $(40 \%)$ belong to one to ten years age group. ${ }^{1}$

The Department of Health (DOH) National Objectives for Health (2011 to 2016) aim to reduce the incidence of dengue fever in the country through intensification of its advocacy campaigns on vector control. This is complemented by risk reduction interventions such as environmental sanitation and removal of mosquito breeding sites. ${ }^{2,10}$ However, the implementation of dengue control program seems to be facing tough challenges as indicated by the increasing number of dengue cases for the last five years. ${ }^{1,10}$ Changing weather patterns, geography, living conditions, habits and behaviors of people, as well as mosquito vector characteristics, further strain the current prevention and control measures. ${ }^{3}$

The Department of Science and Technology (DOST) as a response to this public health problem has supported the development of a novel technology which aims to control the population of dengue virus-carrying mosquitoes. The Ovicidal-Larvicidal (OL) trap consists of a $5 \times 2.5 \times 3.5$ inch in diameter container or tumbler, black in color to attract egg-laying female mosquitoes, with a $6.5 \times 1$ inch lawanit strip placed inside for mosquitoes to lay their eggs on, and a solution which is lethal to both ova and larvae (Figure 1). 4,5 Initial field testing of the OL trap system with pelletized extracts of Piper nigrum L. for Aedes mosquitoes in Quezon City and Marikina City has shown a decrease in dengue cases. ${ }^{4,6}$ Thereafter, the OL traps have been distributed to dengue hotspots by the respective Local Government Units (LGU's) and yielded similar results. The DOH has fostered partnerships with more LGU's in the distribution of the OL traps to their constituents. ${ }^{4}$ Part and parcel of the distribution mechanism is the training of DOST and DOH staff, including barangay health workers (BHW's) on the 
proper distribution, use and maintenance of OL traps at the household level. The BHW's, being at the frontline of health service delivery, were tapped to distribute, monitor and collect OL traps from the recipient households after 5 to 7 days. ${ }^{4}$

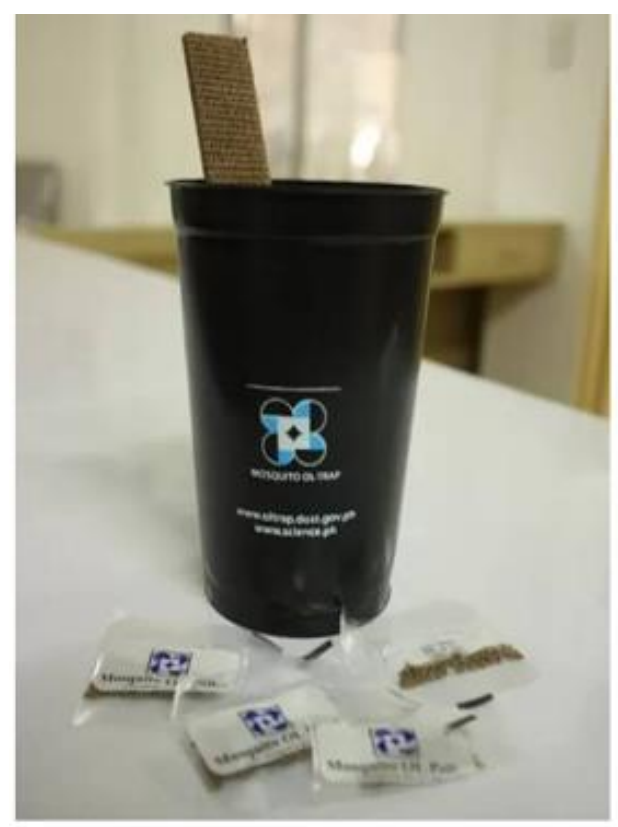

Figure 1. DOST Mosquito Ovicidal-Larvicidal (OL) Trap. ${ }^{5}$

Studies on the assessment and filling of gaps of the antiDengue Program in the Philippines and on knowledge about OL Trap use found that majority of the respondents are well informed regarding Dengue and its prevention and management. According to authors, however, many respondents were uncertain whether Ovitraps lessen the number of dengue cases. This may be because the respondents are not aware of what an Ovitrap is..$^{9,10}$

Apart from the above literature, there is a dearth of local published studies which assess the psychosocial factors that influence acceptance of OL traps among users in the communities. It is for this reason that this study was undertaken, specifically assessing respondents' 1) knowledge on the components and mechanism of action of the OL Traps, its installation and maintenance, 2) attitudes towards its use and maintenance, 3) perceptions on its effectiveness and market feasibility, and practices related to its installation and maintenance for dengue mosquito vector control. The results of the study can be used as basis for the development of an educational intervention to increase acceptance of OL traps at the local level.

\section{Methods}

\section{Research Design}

This study used a descriptive cross-sectional study design to determine the knowledge, attitudes, perceptions and practices of residents on the effectiveness of the novel OL traps in reducing the population of dengue mosquito vectors.

\section{Study Site}

The study was done in one barangay in Quezon City. ${ }^{7}$ It was selected as it was one of the barangays with the highest reported dengue cases in 2011 and one of the first barangays which received OL traps from the DOST. As of 2007, its recorded population was 56,620 with approximately 7,771 households. ${ }^{8}$ It has 5 depressed areas, which, except for one, are flood-prone.

\section{Study Population}

Study participants included residents of the selected barangay, aged 13 to 82 years old, living in a household, physically able to take part in the interview, gave written consent, and previously received and kept an OL trap and related instructions from 5 to 7 days prior the study. In households whose recipients of OL traps and instructions were minors but complied with the other inclusion criteria, were also included in the study.

\section{Sample Size}

Sample size of 383 was calculated using 0.47 as an estimate of true proportion with a $95 \%$ level of confidence and a maximum permissible error of 0.05 . The actual study yielded a total of 399 respondents.

\section{Sample Recruitment}

Respondents were recruited through purposive sampling using the following criteria: provision of informed consent, ability to understand and respond during the interview, recipient of OL traps, the OL traps received were kept for five to seven days prior to the study.

\section{Method of Data Collection}

The study employed face-to-face interviews using a pilot-tested interview schedule. The survey tool included questions on knowledge, attitudes and perceptions related to components, properties, installation and maintenance OL traps. The interview took an average of 15 minutes to complete. Attitudes were assessed using a four-point Likert Scale, an enlarged version of which was shown to the respondents to facilitate understanding and choice selection. To ascertain practices related to the use of OL traps, an actual observation was done among households that still maintained the OL traps at the time of the interview. The interviewers were trained on proper interviewing to minimize inter-observer variability.

\section{Data Processing and Analysis}

Data were encoded and analyzed using Epi Info ${ }^{\mathrm{TM}}$ Version 3.5.3. Accuracy of data entry was validated by the members of the study team. Except for age, data on socio- 
demographic profile were presented as proportions. Data on knowledge (categorized as correct or incorrect), attitudes (measured using strongly agree - strongly disagree continuum), perceptions and practices related to OL traps were presented as proportions.

\section{Ethical Consideration}

The study was done in accordance with the principles provided for in the General Ethical Guidelines for Health Research. The proposal was approved by the Ethics Review Board of the College of Public Health, University of the Philippines Manila. Written informed consent was obtained from the respondents or legal guardians of respondents who are minor. Apart from the consent, a written assent was also sought from the two respondents who are minor.

\section{Results}

\section{Socio-demographic data}

A total of 399 residents were interviewed in the study. Majority of the respondents (92.2\%) were females, the age ranged from 13 to 82 with a mean of $39.2+/-10$. There were two respondents $(0.5 \%)$ who were minor, i.e. 13 and 16 years old. Most respondents (85\%) were married and more than half $(54 \%)$ attained secondary education.

\section{Knowledge related to the components of OL traps}

Table 1 shows that more than $93 \%$ of the respondents had correct knowledge on the color and components of the OL trap container. Two-thirds (66.8\%) knew that the purpose of the "lawanit" is to provide a site for female egglaying. However, only $44 \%$ knew that it was the water solution in the OL trap which was lethal to mosquito eggs.

Table 1. Distribution of respondents according to their knowledge of the components of OL traps, Bgy. Gulod, Quezon City, 2012, N=399.

\begin{tabular}{lcc}
\hline Knowledge of Components of the OL traps* & \multicolumn{2}{c}{ Correct Response } \\
\cline { 2 - 3 } & Freq. & $\%$ \\
\hline $\begin{array}{l}\text { Correct color of the OL Trap container } \\
\text { OL Trap is made of either a black tin can or cup with a }\end{array}$ & 368 & 96.74 \\
$\begin{array}{l}\text { strip of lawanit. } \\
\text { The purpose of the lawanit strip is to provide a site for } \\
\text { the female Aedes mosquito to lay its eggs on. }\end{array}$ & 251 & 63.64 \\
$\begin{array}{l}\text { The solution (water with ovicide and larvicide) is the } \\
\text { component of the OL Trap that is deadly to mosquito } \\
\text { eggs }\end{array}$ & 177 & 44.36 \\
\hline *multiple response & & \\
\end{tabular}

Knowledge related to the installation and maintenance of the $O L$ traps

Correct installation and maintenance of the OL trap is crucial in achieving optimal results, therefore, determining the respondents' knowledge on these aspects is deemed necessary. A very high proportion of the respondents (96\%) knew that OL traps should be placed in low-lying as well as in dark areas, and that it is important that they were protected from exposure to sunlight and rain fall $(91.47 \%)$ (Table 2).

Table 2. Distribution of respondents according to their knowledge of the installation and maintenance of the OL Trap, Bgy. Gulod, Quezon City, 2012, N=399.

\begin{tabular}{lcc}
\hline \multirow{2}{*}{ Knowledge on Installation and Maintenance* } & \multicolumn{2}{c}{ Correct Knowledge } \\
\cline { 2 - 3 } & Freq. & $\%$ \\
\hline OL traps should be placed in low-lying areas. & 383 & 95.99 \\
OL traps should be placed in dark areas. & 383 & 95.99 \\
It is important to protect the OL traps from both the & 354 & 91.47 \\
sun and rain. & & \\
$\begin{array}{l}\text { Two OL traps per household are enough to effectively } \\
\text { reduce mosquito populations; one placed indoors, and }\end{array}$ & 228 & 59.53 \\
$\begin{array}{l}\text { the other, outdoors. } \\
\text { Only one pack of the OL pellets that should be used }\end{array}$ & 219 & 57.48 \\
$\begin{array}{l}\text { Which side of the lawanit strip (rough or smooth) } \\
\text { should be facing up when the OL trap is installed }\end{array}$ & 191 & 53.35 \\
\hline *multiple response & & \\
\end{tabular}

More than half $(57.5 \%)$ said that only one pack of OL pellets should be used per container; $53.4 \%$ knew that the rough side of the lawanit strip should be facing up during installation.

Table 3. Distribution of respondents according to their knowledge related to the mechanism of action of the OL Trap, Bgy. Gulod, Quezon City, 2012, N=399

\begin{tabular}{lcc}
\hline Knowledge on Mechanisms of the OL traps* & \multicolumn{2}{c}{ Correct Knowledge } \\
\cline { 2 - 3 } & Freq. & $\%$ \\
\hline $\begin{array}{l}\text { The solution itself is an ideal site for the female Aedes } \\
\text { mosquito to lay its eggs on. }\end{array}$ & 277 & 71 \\
$\begin{array}{l}\text { OL traps are composed of toxic materials which could } \\
\text { harm me and my family }\end{array}$ & 271 & 67.6 \\
$\begin{array}{l}\text { Only the dengue-carrying mosquitoes can be killed by } \\
\text { the OL traps. }\end{array}$ & 175 & 46.1 \\
$\begin{array}{l}\text { OL traps kill any kind of mosquito } \\
\text { Female mosquitoes are attracted by the scent of the OL }\end{array}$ & 120 & 35.3 \\
$\begin{array}{l}\text { Trap. } \\
\text { OL traps can kill mosquito eggs even without the OL }\end{array}$ & 111 & 27.8 \\
formulation. & & \\
The presence of live larvae is an indication that the OL \\
$\begin{array}{l}\text { Trap is not working. } \\
\text { OL traps kill both eggs and larval stages of mosquitoes }\end{array}$ & 60 & 22.6 \\
\hline *multiple response & & \\
\end{tabular}

\section{Knowledge related to the mechanism of action of the OL traps}

Respondents were also asked about their knowledge on the mechanism involved in the OL traps. Table 3 shows that more than two-thirds $(71.0 \%)$ were correct in saying that the solution itself is an ideal medium for egg laying. However, $68 \%$ also thought that OL traps are composed of toxic materials which could harm oneself and one's family. Only $35 \%$ had correct knowledge on the following: OL traps can kill any kind of mosquitoes and that female mosquitoes are attracted by the scent of the OL traps. It is noteworthy that $28 \%$ mentioned that OL traps can kill without the OL formulation. About $23 \%$ said that presence of live larvae in 
the container is an indication that the trap did not work. Only $16.3 \%$ was correct in saying that OL traps kill both mosquito eggs and larvae.

\section{Attitudes towards the use and maintenance of $O L$ traps}

Table 4 shows the distribution of respondents' attitudes toward statements which pertain to the use and maintenance of OL traps. It can be seen that a very high proportion agree that OL traps can aid in the control of dengue $(92.4 \%)$ and that it can be an effective way of controlling dengue (88.2\%).

Almost all (96.2\%) respondents believe that elimination of breeding places is a primary means of preventing dengue. Two-thirds (65\%) expressed that they can prevent dengue without the use of OL traps. Almost half $(48 \%)$ answered that using OL traps is time consuming and very complicated. It is noteworthy that only $56.9 \%$ believe that using OL traps alone will significantly decrease the chance of one's family in contracting dengue fever.

Table 4. Distribution of respondents according to their attitudes towards the use and maintenance of OL traps, $\mathrm{N}=399$

\begin{tabular}{lcc}
\hline $\begin{array}{l}\text { Attitudes towards the use and maintenance of OL } \\
\text { traps }\end{array}$ & Agree & Disagree \\
\hline $\begin{array}{l}\text { Elimination of breeding places as a primary means } \\
\text { of prevention }\end{array}$ & $96.20 \%$ & $3.80 \%$ \\
$\begin{array}{l}\text { I can aid in the prevention and control of dengue } \\
\text { through the use of OL traps }\end{array}$ & $92.40 \%$ & $7.50 \%$ \\
$\begin{array}{l}\text { OL traps can be an effective way of controlling the } \\
\text { increasing incidence of dengue }\end{array}$ & $11.80 \%$ \\
$\begin{array}{l}\text { I can prevent Dengue without the use of OL traps } \\
\text { The use of OL traps alone will significantly } \\
\text { decrease the chance of my family contracting } \\
\text { dengue }\end{array}$ & $56.90 \%$ & $45.00 \%$ \\
$\begin{array}{l}\text { The use of OL traps is time-consuming and very } \\
\text { complicated }\end{array}$ & $48.20 \%$ & $51.80 \%$ \\
\hline
\end{tabular}

Perceptions on the effectiveness and market feasibility of the $O L$ traps

Despite its proven effectiveness, OL traps as a novel technology may pose some problems towards its use. Hence, it is important to assess the acceptability and willingness of respondents to purchase and use the device. Respondents were asked on their perceptions of the effectiveness and market feasibility of the OL traps. Table 5 shows that a very high proportion believed that OL trap is a necessity in every household (83\%) and that they are willing to buy them once they become commercially available (81.7\%). The respondents were willing to spend an average amount of PhP 51.08 per OL trap. Seventy nine percent believed on the significant effect of OL traps in the reduction of dengue cases. Almost three-fourths (73.4\%) believed, based on their experience, that OL traps are effective in controlling mosquito populations. More than a third (34.8\%) answered that OL traps could replace mosquito coils and nets as mosquito control measures.
Table 5. Distribution of respondents' perceptions on the effectiveness and market feasibility of the OL traps, N = 399

\begin{tabular}{|c|c|c|c|c|c|c|}
\hline \multirow{2}{*}{$\begin{array}{l}\text { Perceptions on the } \\
\text { effectiveness* }\end{array}$} & \multicolumn{2}{|c|}{ YES } & \multicolumn{2}{|c|}{ NO } & \multicolumn{2}{|c|}{ UNSURE } \\
\hline & Freq. & $\%$ & Freq. & $\%$ & Freq. & $\%$ \\
\hline $\begin{array}{l}\text { OL traps are a necessity } \\
\text { in every household }\end{array}$ & 331 & 83 & 63 & 15.7 & 5 & 1.3 \\
\hline $\begin{array}{l}\text { Willing to buy OL traps } \\
\text { should they become } \\
\text { commercially available }\end{array}$ & 326 & 81.7 & 70 & 17.5 & 3 & 0.8 \\
\hline $\begin{array}{l}\text { OL traps had significant } \\
\text { effect in the reduction of } \\
\text { dengue cases }\end{array}$ & 315 & 79 & 63 & 15.7 & 21 & 5.3 \\
\hline $\begin{array}{l}\text { Based on experience, OL } \\
\text { traps are effective in the } \\
\text { control of mosquito } \\
\text { populations }\end{array}$ & 293 & 73.4 & 87 & 21.8 & 19 & 4.8 \\
\hline $\begin{array}{l}\text { OL traps can replace the } \\
\text { use of the conventional } \\
\text { mosquito coils and nets }\end{array}$ & 139 & 34.8 & 251 & 62.9 & 9 & 2.3 \\
\hline
\end{tabular}

Practices related to installation and maintenance of the OL traps

To establish the practices of the household on the use of OL traps, an actual observation of how the OL traps were installed and maintained was undertaken. Thirty (30) households where OL traps were installed during the interview were included in the observation. Table 6 shows that the observed households had correct practices as far as the installation and maintenance of OL traps were concerned. However, only $50 \%$ were noted to have correctly used the lawanit strip (50\%) while $46.7 \%$ maintained the correct water level (i.e., up to DOST line in the black container).

Table 6. Distribution of respondents' practices related to installation and maintenance of the OL traps as observed during the interviews, $\mathrm{n}=30$

\begin{tabular}{lcc}
\hline \multicolumn{1}{c}{ Practices* $^{*}$} & \multicolumn{2}{c}{ YES } \\
\cline { 2 - 3 } & Freq. & $\%$ \\
\hline OL Trap was placed in low lying areas & 30 & 100 \\
OL Trap was placed in dark areas & 29 & 96.7 \\
OL Trap was placed in an area protected from the rain & 28 & 93.3 \\
OL Trap was placed away from sunlight & 27 & 90 \\
OL traps used was correct, i.e. black/dark colored & 27 & 90 \\
container & & \\
One pack of pellets was used for the OL traps solution & 24 & 80 \\
The rough side of the lawanit strip faced up & 15 & 50 \\
The water level was maintained correctly, i.e. up to the & 14 & 46.7 \\
DOST line & & \\
\hline *multiple response & \multicolumn{2}{c}{} \\
\end{tabular}

Moreover, the respondents' perceptions on the advantages and disadvantages of OL traps were also sought. The most common advantages cited were: OL traps lessen the number of mosquito population (20\%), these can help them avoid dengue infection (11.9\%). 14.7\% did not see any advantages. On the other hand, the most frequently mentioned disadvantage of using OL traps included the perception that they are hazardous to children $(21.5 \%)$, they 
are poisonous $(9 \%)$, and that they need to be monitored (5\%). Almost half $(44 \%)$ said that OL traps had no disadvantages and 13\% did not know the answer.

\section{Discussion}

The sample population, in general, had good knowledge, favorable attitudes, good perceptions and correct practices as far as the components, properties, installation, maintenance, mechanisms involved, and effectiveness of the OL traps. Specifically, respondents were generally knowledgeable on the components of the OL traps including its color and make-up, its proper installation so that they will be protected from environmental factors like sunlight, rainfall and children's reach. The respondents believed that OL traps are effective in decreasing mosquito population ${ }^{9}$ and that they felt this technology is a necessity in every household. In fact, most respondents expressed willingness to purchase the OL traps once they become commercially available. It is interesting to note that respondents claimed that they were willing to spend, on the average, PhP 51.00 per OL trap, which is much higher compared to the actual commercial price of PhP 25.00-30.00 and the PhP 15.00 cost in Carandang's study. A high level of knowledge on the effectiveness of OL trap was supported by Carandang's study, in which most of his respondents knew how it works, believed that OL trap was effective and that they would recommend its use, ${ }^{9}$ however, they need to be taught on how to properly dispose the solution containing mosquito eggs. This result seems to differ from Solidum's findings that many of his respondents were uncertain whether Ovitraps can lessen the number of dengue mosquito vectors. However, it should be noted that the respondents from Solidum's study might be unclear whether the Ovitraps being mentioned contain the same solution that has both ovicidal and larvicidal properties present in the OL traps of DOST. Respondents from his study would, nevertheless, recommend its use. Most respondents in the same study also said that the $\mathrm{PhP} 15.00$ cost of one OL trap was reasonable. ${ }^{9}$ This is much lower compared to the amount that respondents in this present study were willing to pay.

The high level of knowledge could be attributed to information dissemination efforts given by the health workers during distribution of the OL traps as well as the influence of mass media ${ }^{10}$ more especially during dengue epidemic. The barangay, through the BHWs actually explained the components, mechanism involved, how to maintain and monitor the OL traps before these are issued to residents. ${ }^{10}$

A high proportion of respondents in this study thought that two OL traps to be installed per household are enough to effectively reduce mosquito population, this deviates from the recommendation by the Industrial Technology Development Institute (ITDI). The institute specifies that each household should have at least four OL traps, two placed indoors and another two outdoors. ${ }^{4}$ The discrepancy could probably be due to the limited number of OL traps distributed to the community for economic reasons, hence, only two were issued per household.

There were issues related to the mechanisms involved in the OL traps which need to be further addressed since they can influence greater community acceptance. The misconception that OL traps can kill mosquito eggs even without the pellet formulation included was high. This was supported by the finding that only $44 \%$ of the respondents thought that it was the solution which is deadly to dengue mosquito eggs and larvae. This could be partly due to the inadequate explanation by the health workers or difference in the manner by which OL traps were installed. In some cases, BHWs themselves prepared and installed the traps, whereas in others it was the family members who actually placed the pellets. The above finding is critical because if the community thinks that OL traps can kill mosquito eggs even without adding the formulation then this may result to noninclusion of pellets. Ultimately, OL traps without the formulation could serve as additional breeding sites rather than a mosquito vector control measure. Pellets contain extracts that are supposedly responsible for killing mosquito eggs and larvae in the OL traps. ${ }^{4}$

Knowledge on the number of packs of OL pellets that should be used in one preparation needs to be further improved. There was moderate knowledge regarding which side of the lawanit should be facing up during installation, although this may not be very crucial as experts opined that Aedes mosquitoes will lay their eggs on the side of the container that touches water (personal communication with Dr. Lilian delas Llagas, 2012).

Almost a quarter mentioned that presence of live larvae in the container is an indication that the OL traps is not working. The OL trap is supposed to be effective against Aedes mosquito eggs and larvae, however, field testing results showed that larval mortality is only from 83 to $89 \%{ }^{3}$ During the interviews, there were respondents who complained that they noticed emerging larvae despite adding the biocidal component.

It is good to note that a very high proportion of respondents expressed a positive attitude towards the use and maintenance of OL traps. Residents recognized the important role they have in dengue mosquito control by using OL traps, and they also believe in the effectiveness of OL traps in curbing mosquito population and eventually incidence of dengue. However, it is alarming to find that two-thirds also believe that OL traps are composed of toxic materials which could harm oneself and his/her family. This is supported by respondents who mentioned that OL traps are either hazardous to children or poisonous. These findings could be explained partly by the following: it might have not been adequately stressed in the information 
campaign or respondents may have misinterpreted the message that OL traps should be kept away from children's reach giving an impression that it contains materials which could be harmful to children. Another possibility is that because the solution in the OL traps is lethal to mosquito eggs and larvae, hence, it contains ingredients which are toxic to humans. It should be stressed here that the solution consists of black pepper seeds which is lethal to mosquito eggs but harmless to humans. ${ }^{4,5}$

While almost everybody believes that elimination of breeding places is the primary means of preventing dengue, more than half still had an incorrect notion that using OL traps alone will significantly decrease the family's chance of contracting dengue fever. This is corroborated by 35\% who thought that OL traps can replace other traditional control measures like mosquito coils and nets. It should be emphasized that controlling mosquito population requires an integrated approach and OL traps is just one of the innovative strategies which only targets immature mosquitoes. Implementing risk reduction strategies which include environmental sanitation and removal of mosquito breeding sites on top of information dissemination, early diagnosis and quality clinical care should still be the mainstay of any prevention and control strategies and with OL traps as a very important complement. ${ }^{2}$

The belief that using OL traps is time-consuming and very complicated may negatively affect possible community adoption of the technology. This belief runs counter to the DOST claim that the OL trap process is simple. . $^{4,5}$

It was also revealed that $80 \%$ to $100 \%$ of respondents included in the observation had good practice in terms of proper installation, use and maintenance of OL traps. Only half positioned the correct side of the lawanit during installation which could be because of inadequate explanation and misunderstanding during the orientation. This incorrect practice is still acceptable because dengue mosquitoes, just like in the regular ovitraps, still lay their eggs on the side of the container close to the water level.

\section{Conclusions}

In summary, the results of this cross-sectional study provides important information on the knowledge, attitudes, perceptions and practices relevant to the OL trap, and may be used as basis to improve the National Dengue Control Program. The study established that good knowledge, positive attitudes and perceptions of the initial beneficiaries of this novel technology are consistent with correct practices related to OL traps.

\section{Recommendations}

To increase the acceptability of the OL traps at the household level, it was found that there were misconceptions that need to be corrected. It is recommended that BHWs be trained on delivering a standard, clear and concise message regarding the use of each component of the
OL trap. The message should include the safety issues, proper maintenance, correct number of OL traps per household, and what to do with OL traps after 5 to 7 days. Comparison of the knowledge, attitudes, perceptions and practices of the respondents with important variables, such as sociodemographic variables, can be done in future studies.

\section{Limitations}

The above-mentioned conclusion and recommendations should be taken in the light of study's possible limitations. The stratified random sampling that was initially planned was changed to purposive sampling because not all households received the OL traps (one of the study's inclusion criteria). The purposive nature of sampling may affect the generalizability of the results. There were two respondents $(2 / 299$ or $0.5 \%)$ who were minor who were included in the study simply because they were the ones who received the OL traps and the instructions. Only $8 \%$ (30/399) of the respondents were included in the observation because they were the only ones who still had OL traps installed at the time of the interview. For the rest of the respondents (92\%), the OL traps were already collected by the BHWs, hence, its installation and maintenance could no longer be observed. This may or may not represent the OL trap-related behavior of the greater majority of the sample. The study only aimed to describe the knowledge, attitudes, perceptions and practices of the respondents related to OL traps.

\section{References}

1. Department of Health National Epidemiology Center, Public Health Surveillance and Informatics Division, Disease Surveillance Report, Morbidity Week 36, September 1 to 7, 2013.

2. 2011-2016 National Objectives for Health, Health Sector Reform Agenda Monographs. Manila, Republic of the Philippines - Department of Health, 2011 (DOH HSRA Monograph No. 12).

3. de las Llagas LA. Biology and control of Aedes mosquito vectors of Dengue/Dengue Haemorraghic Fever in the Philippines. UP Manila Journal. 1995; 1:11-13.

4. DOST Mosquito Ovicidal/Larvicidal (OL) Trap for Dengue Prevention [Online]. 2014 [cited 2014 May] Available from http://www.filipinoinventionsanddiscoveries.blogspot.com.

5. Frequently asked questions on the DOST Mosquito Ovicidal-Larvicidal Traps [Online]. 2014 [cited 2014 May]. Available from OLTrap.blogspot.com.

6. Briones AV, Garbo AG, Casa EP, Bion HH, Almanzor NE, Bernardo ST. Field testing of ovicidal-larvicidal trap system with pelletized extracts of Piper nigrum L. for Aedes mosquito in Quezon City and Marikina City. Acta Med Philipp. 2013; 47:36.

7. Wikimapia. Barangay X Map Coordinates [Online]. 2011 [cited 2011 July] Available from http://wikimapia.org.

8. National Statistics Office (NSO) [Online]. 2011 [cited 2011 October]. Available from http://www.census.gov.ph/content/2010-census-population-andhousing-reveals-philippine-population-9234-million.

9. Carandang AP, Anonas FV, Lazcaro JM. Knowledge of OL Trap users on mosquito, dengue, and OL trap as basis for comics development to promote OL trap use. Transactions National Academy of Science and Technology. 2013; 35:1.

10. Solidum JN, Solidum GG. Official Conference Proceedings of the the Asian Conference on the Social Sciences 2013 [Online]. 2014 [cited 2014 May]. Available from http://www.iafor.org. 